\title{
Case Report "A Dissecting Aneurism of the Aortic Arch at the Left Subclavian Origin Extending to Descending Thoracic and Abdominal Aorta with False and True Lumens"
}

\author{
Juna Musa ${ }^{1 *}$, Loran Rakovica ${ }^{2}$, Fjolla Hyseni ${ }^{3}$, Ali Guy ${ }^{4}$, Mina Mikel ${ }^{5}$, \\ Masum Rrahman ${ }^{6}$, Marsela Ceno ${ }^{7}$, Valon Vokshi ${ }^{8}$, Viola Sulollari ${ }^{9}$, \\ Ineida Boshnjaku ${ }^{9}$ and Nosehy Yousef ${ }^{10}$ \\ ${ }^{1}$ Postdoctoral Research Fellow, Department of Surgery, Critical Care and Trauma, \\ Mayo Clinic, Rochester Minnesota, USA \\ ${ }^{2}$ Medical Doctor, Faculty of Medicine, Prishtina, Kosovo \\ ${ }^{3}$ Department of Urology, NYU Langone Health, New York, USA \\ ${ }^{4}$ Assistant Professor, Department of Physical Medicine and Rehabilitation, New York \\ University School of Medicine, NYU Medical Center, New York, USA \\ ${ }^{5}$ Department of Internal Medicine Registrar, Jaber Al-Ahmed Hospital, Kuwait \\ ${ }^{6}$ Department of Neurosurgery, Mayo Clinic, Rochester, MN-55905, USA \\ ${ }^{7}$ Department of Surgery, Klinikum Mittelbaden Baden Baden, Germany \\ ${ }^{8}$ Department of Anesthesiology, University Clinical Center Kosovo \\ ${ }^{9}$ Medical Doctor, Hospital Mother Teresa, Tirane, Albania \\ ${ }^{10}$ Department of Cardiology, El Mabarrah Hospital Health Insurance Organization, \\ Egypt \\ *Corresponding Author: Juna Musa, Postdoctoral Research Fellow, Department \\ of Surgery, Critical Care and Trauma, Mayo Clinic, Rochester Minnesota, USA.
}

Received: August 17, 2020

Published: September 17, 2020

(C) All rights are reserved by Juna Musa., et al.

\begin{abstract}
Aneurysm of the descending thoracic (DTA) and thoraco-abdominal aorta (TAA) is a life-threatening disorder given the risks of aortic dissection which requires quick and accurate diagnosis. However, correct diagnosis of AAD could be difficult in some instances because of the wide variety of clinical presentations. The clinical picture depends on the aortic branches involved, the size and direction of the dissection, the duration of symptoms, and the location of intimae tear.

In this case report, 48 years old male presented with sudden onset of severe interscapular heaviness type of pain of three hours duration, associated with radiation to anterior chest wall and sweating. He is non-smoker but hypertensive. ECG showed sinus rhythm, with ST-depression and T-wave inversion in the infero-lateral leads. Timed multi-slice helical CT acquisition was taken and revealed a dissection flap of the aortic arch beginning nearly at the left subclavian origin extending up to involve the whole descending thoracic and abdominal aorta with false and true lumens. Cardio thoracic surgeon consultation was obtained.
\end{abstract}

Keywords: Descending Aortic Aneurysms; Dissection of the Aorta; ACS-Artery Coronary Syndrome; Ischemia

\section{Introduction}

Aortic dissection is the most common acute emergency condition of the aorta and often has a fatal outcome.

Aortic dissections are classified on the basis of the site of the intimal tear according to the Stanford classification system. Type
A aortic dissection involves the ascending thoracic aorta and may extend into the descending aorta, whereas in a type B dissection the intimal tear is located distal to the left subclavian artery [1].

It has been reported that approximately $14.2-15.7 \%$ of aortic dissections are accompanied by descending aortic aneurysms,

Citation: Juna Musa., et al. "Case Report "A Dissecting Aneurism of the Aortic Arch at the Left Subclavian Origin Extending to Descending Thoracic and Abdominal Aorta with False and True Lumens"'. Acta Scientific Medical Sciences 4.10 (2020): 54-58. 
whereas approximately 1.6 - 4.9\% of descending aortic aneurysms coexist with aortic dissection. Therefore, type B aortic dissection with descending aortic aneurysms occurs rarely [2].

The sudden onset of severe chest or back pain is the most frequent symptom. Anterior chest pain is associated with dissections involving the ascending aorta, while interscapular back pain is associated with descending aortic dissections [3].

While the pain may be confused with that of a heart attack, $\mathrm{AD}$ is usually not associated with the other suggestive signs, such as heart failure and ECG changes. Less common symptoms that may be seen in the setting of $\mathrm{AD}$ include congestive heart failure $(7 \%)$, fainting $(9 \%)$, stroke $(6 \%)$, ischemic peripheral neuropathy, paraplegia, and cardiac arrest. If the $\mathrm{AD}$ involves the abdominal aorta, compromise of one or both renal arteries occurs in 5 - $8 \%$ of cases, while ischemia of the intestines occurs about $3 \%$ of the time $[4,5]$.

The overall outcome is determined by the type and extent of dissection and the presence of associated complications; therefore, evaluation of the entire aorta, branch vessels, and iliac and proximal femoral arteries is recommended to aid in treatment planning [6].

In recent years a special focus has been placed on the involvement of the aortic arch in primary type $B$ dissection. The term nonA non-B dissection addresses both retrograde dissections after a primary tear in the descending aorta as well as a primary tear in the aortic arch. The value of this characterization lies in the very high probability of the necessity for invasive treatment as soon as the aortic arch is affected [7].

\section{Case Report}

Forty-eight-year-old Egyptian male presented with sudden onset of severe interscapular pain of three hours duration that radiated to anterior chest wall associated with sweating. Hypertensive on presentation due to self-discontinuation of medication three years ago. Clinical examination revealed an anxious, pale, sweaty, obese man. His heart rate was 90 beats per minute. All peripheral pulses were palpable, blood pressure was $240 / 140 \mathrm{mmHg}$ on the right hand and 234/136 on the left hand, 194/109 $\mathrm{mmHg}$ on the right leg and 224/116 mmHg on the left leg. Examination of heart, chest and abdomen revealed no changes. Ophthalmoscopic examination revealed grade II hypertensive retinopathy. ECG showed sinus rhythm with ST-depression and T-wave inversion in the infero- lateral leads. Chest X-ray revealed cardiomegaly with prominent aortic knuckle. Cardiac enzymes were normal. Hemoglobin was $16.1 \mathrm{mg} / \mathrm{dl}$ and total white blood cells were $17.1 \times 10^{12} / \mathrm{L}$, with predominant neutrophils and platelets levels of $475 \times 10^{3} / \mathrm{L}$. Coagulation studies and renal function were normal. Patient was admitted to the coronary care unit. Pain was relieved by intravenous morphine. Infusion of nitroglycerin was started. Bisoprolol $10 \mathrm{mg}$, Amlodipine $10 \mathrm{mg}$ were administered orally. Blood pressure lowered to $130 / 75 \mathrm{mmHg}$ within few hours. Echocardiography showed left ventricular hypertrophy with diastolic dysfunction.

Timed multi-slice helical CT acquisition with automatic phase, was taken after stabilizing the vital signs, revealed A dissection flap of the aortic arch beginning nearly at the left subclavian origin extending up to involve the whole descending thoracic and abdominal aorta with false and true lumens.
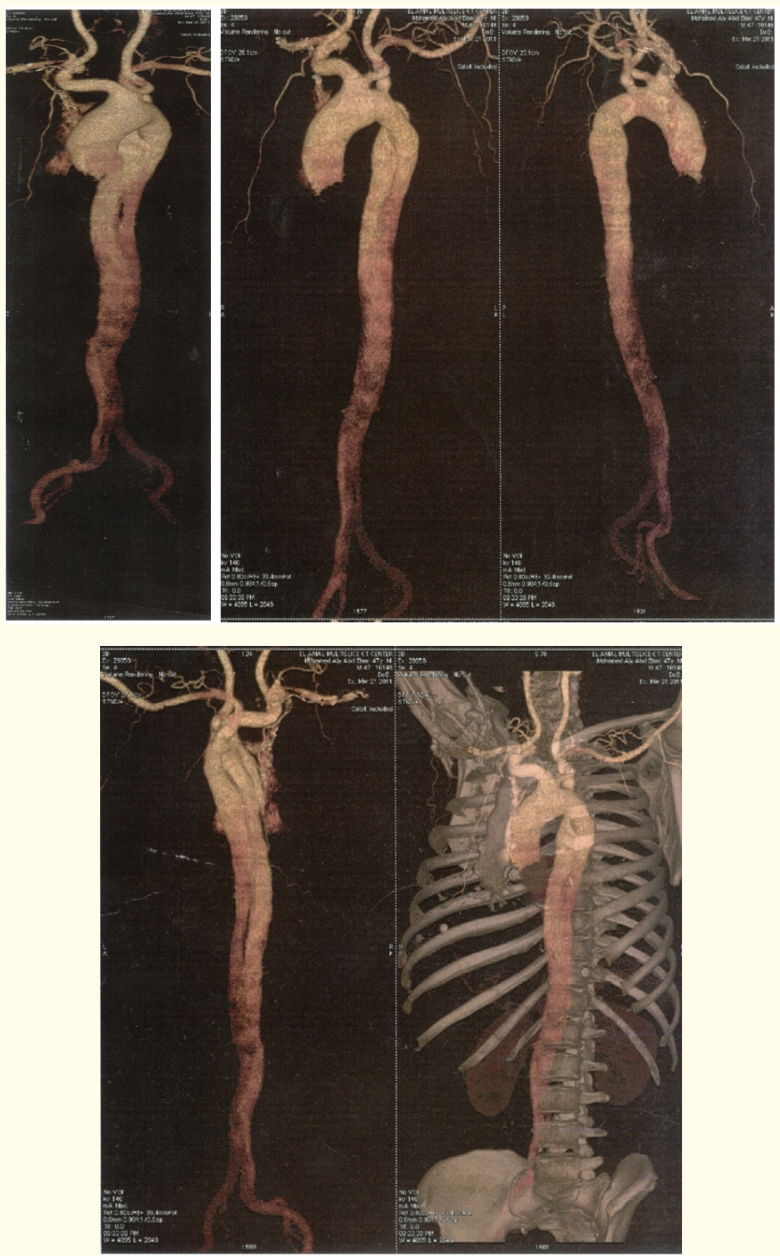

Figures 
The flap extends down to the bifurcation and involves the right common iliac and proximal short segment of right external iliac artery. No definite dissection flap of the left iliac artery and no definite thrombosis could be detected. A cardio-thoracic surgeon consultation was obtained.

\section{Discussion}

Aortic dissections are a subclass of acute aortic pathology characterized by a tear in the innermost layer of the aortic wall, the intima, allowing some of the blood flow to escape from the main passageway (the true lumen) of the aorta and reroute into an artificial secondary passageway (the false lumen) between the intima and media of the aortic wall. Driven by the high pressure within the aorta, the flow of blood through the entry tear leads to separation of the layers of the aortic wall and subsequent propagation of the false lumen either antegrade or retrograde along the aorta or occasionally in both directions. This constellation of events can disrupt normal blood flow resulting in clinically significant malperfusion to vital organs or weakening of the aortic wall with ensuing aortic rupture [1,2].

A high index of suspicion is required to promptly establish the diagnosis of this life-threatening entity, because the symptoms can be variable and may mimic those of more common conditions. In the International Registry for Acute Aortic Dissection, hypertension was the most common predisposing factor (72\%) for aortic dissection, followed by atherosclerosis (31\%), history of cardiac surgery (18\%), Marfan syndrome (5\%), and iatrogenic causes (4\%).

Aortic dissections are classified based on the anatomic distribution of the dissection, the time from symptom onset, and the presence of complications.

The most commonly used classification schemes for acute aortic syndromes are the De Bakey and the Stanford system. For classification purposes, the ascending aorta refers to the part of the aorta proximal to the brachiocephalic artery, and the descending aorta refers to the aorta distal to the left subclavian artery [4-6].

In the population-based Malmö Diet and Cancer Study (MDCS), type A dissections were more frequently (58\%) seen than type B dissections. In the OXVASC study $67.3 \%$ of patients with aortic dissection had arterial hypertension and $61.5 \%$ of patients were smokers. Smoking and arterial hypertension were also significant risk factors for developing aortic dissection in the MDCS study, in addition to increasing age, male gender, and low apolipoprotein A-1 levels.

It has been reported that approximately $14.2-15.7 \%$ of aortic dissections are accompanied by descending aortic aneurysms, whereas approximately $1.6-4.9 \%$ of descending aortic aneurysms coexist with aortic dissection Therefore, type B aortic dissection with descending aortic aneurysms occurs rarely [7].

In this case, our patient was typically hypertensive male, non smoker with a sudden onset of severe interscapular heaviness type of pain, associated with radiation to anterior chest wall due to myocardial ischemia caused by a dissection flap of the aortic arch beginning nearly at the left subclavian origin extending up to involve the whole descending thoracic and abdominal aorta with false and true lumens.

Chest pain is the most frequent symptom of acute AD. Abrupt onset of severe chest and/or back pain is the most typical feature. The pain may be sharp, ripping, tearing, knife-like, and typically different from other causes of chest pain; the abruptness of its onset is the most specific characteristic. The most common site of pain is the chest (80\%), while back and abdominal pain are experienced in $40 \%$ and $25 \%$ of patients, respectively. Anterior chest pain is more commonly associated with Type A AD, whereas patients with Type B dissection present more frequently with pain in the back or abdomen.

Myocardial ischemia or infarction may be present in $10-15 \%$ of patients with $\mathrm{AD}$ and may result from aortic FL expansion, with subsequent compression or obliteration of coronary ostia or the propagation of the dissection process into the coronary tree. In the presence of a complete coronary obstruction, the ECG may show ST-segment elevation myocardial infarction. Also, myocardial ischemia may be exacerbated by acute aortic regurgitation, hypertension or hypotension, and shock in patients with or without preexisting coronary artery disease. This may explain the observation that approximately $10 \%$ of patients presenting with acute Type B AD have ECG signs of myocardial ischemia as it was found in our patient [8].

The initial aggressive medical treatment aims at the reduction of the systolic BP and left ventricular ejection fraction. IV antihypertensive treatment should be started emergently in all patients, except in those with hypotension, as soon as the diagnosis of acute aor- 
tic dissection is suspected. The aims of the medical therapy are to reduce the force of the left ventricular contractions, to decrease the steepness of the rise of the aortic pulse wave (i.e. $\mathrm{dP} / \mathrm{dt}$ ), and to reduce the systemic arterial pressure to as low a level as possible without compromising perfusion to the vital organs. At the present time, a combination of a $\beta$-blocker and a vasodilator (i.e. sodium nitroprusside) is a standard medical therapy used in patients with aortic dissections [9].

The main purpose of imaging in AAD is the comprehensive assessment of the entire aorta, including the aortic diameters, shape and extent of a dissection membrane, the involvement in a dissection process of the aortic valve, aortic branches, the relationship with adjacent structures, and the presence of mural thrombus.

The early and accurate diagnosis is essential for choosing the appropriate surgical or medical interventions to reduce the high lethality, which is around $1 \%$ per hour after symptom onset in untreated patients.

Both chest X-ray and 12-lead electrocardiogram (ECG) may be helpful in the evaluation of suspected aortic dissection, but a substantial number of patients do not show any abnormalities in these examinations (12 and 31\%, respectively).

According to the International Registry of Acute Aortic Dissection (IRAD) study, ECG alterations suggestive of myocardial ischaemia are likely to delay the correct diagnosis of aortic dissection [10].

The gold standard imaging modality for the diagnosis of AD is computed tomography angiography (CTA). A computed tomography angiogram quickly demonstrates the presence of a dissection flap and allows for visualizing the extent of the dissection and identifying potential areas of malperfusion. CTA is fast, sensitive, and available at most medical centers. If CTA is contraindicated because of contrast-induced allergy or renal disease, transesophageal echocardiography (TEE) or transthoracic echocardiography (TTE) can alternatively be used. A third imaging modality, magnetic resonance imaging (MRI), is likely to provide clinicians with information similar to that provided by CTA. However, MRI has not been widely used to diagnose acute aortic dissection because of lack of access and availability [11]

Treatment of type A dissection typically requires immediate surgery, irrespective of the patient's clinical condition, whereas for type B dissection, endovascular or surgical methods are typically reserved for patients presenting with aortic aneurysms or clinical complications.

For patients with acute aortic dissection (AAD) presenting with the proximal entry tear (ET) in the arch, most current guidelines suggest either surgical repair, or remain inconclusive on the optimal strategy. For AAD with arch ET and involvement of the ascending aorta, an aggressive surgical approach, even including total arch replacement in the acute setting, is usually preferred. However, when there is no ascending aorta involvement, immediate surgery would be recommended by some, if feasible and the patient is viable, whereas others would select medical management if the patient has only an arch dissection without proximal extension, malperfusion, or bleeding, as long as repeat imaging demonstrates stability. In current practice, aortic centers frequently approach AAD with ET in the arch with medical therapy in the absence of ascending aorta involvement. Such patients, sometimes characterized as "non-A, non-B aortic dissection, are treated as a type B dissection [12].

Over the last two decades, thoracic endovascular aortic repair (TEVAR) has emerged as an alternative to open surgical repair for treatment of complicated type B dissection. Various societal guidelines now unanimously propose TEVAR as the treatment of choice for management of complicated disease based on its relatively low morbidity and mortality compared to open surgical outcomes. As the consensually acknowledged standard of care for management of patients with complicated lesions, TEVAR is currently being considered for select patients with initially uncomplicated dissection to mitigate the risks of mid and late-term disease progression and aorticrelated mortality.

A follow-up CT scan usually done prior to hospital discharge or at one month after discharge is a good examination to confirm complete sealing and false lumen thrombosis over the length of the stent-graft in the thorax. TEVAR in acute aortic dissection initially leads to false lumen thrombosis after stent-graft placement that progress from proximal to distal within the thoracic false lumen. Gradually within the year after TEVAR, thrombus resolves and true lumen expansion progresses. CT angiography surveillance examinations of the entire aorta are typically performed at 3, 6, and 12 months, and yearly thereafter to monitor the extent of the dissection, to assess the diameters of the true and false lumens, and to check the perfusion status of the false lumen and the branch vessels [13]. 


\section{Conclusion}

Aortic dissection is the most common acute emergency condition of the aorta and often leads to the patient's death. Aortic arch dissections are uncommon but clinically important abnormalities. The clinical presentation of an acute aortic dissection is often atypical and mimics other common disease processes. Because of the varying symptoms of aortic dissection, the diagnosis is sometimes difficult to make, and largely depend on the extent of the aortic dissection and the presence or absence of malperfusion. Early recognition and prompt operative intervention are crucial for patient survival.

It is recommended that the scanning field include the entire aorta and pelvic vessels to help determine the type and extent of dissection and to help identify relevant complications that may have an impact on surgical planning or management.

\section{Bibliography}

1. Joon Bum Kim., et al. "Risk of Rupture or Dissection in Descending Thoracic Aortic Aneurysm". Circulation 132.17 (2015): 1620-1629.

2. Daniel B Alfson and Sung W Ham. "Type B Aortic Dissections”. Cardiology Clinics 35.3 (2017): 387-410.

3. Azeem S Sheikh., et al. "Acute Aortic Syndrome". Circulation 128.10 (2013): 1122-1127.

4. Kazumasa Orihashi. "Mesenteric ischemia in acute aortic dissection”. Surgery Today 46.5 (2016): 509-516.

5. Arnoud V Kamman., et al. "Visceral Malperfusion in Aortic Dissection: The Michigan Experience". Seminars in Thoracic and Cardiovascular Surgery 29.2 (2017): 173-178.

6. Christoph A Nienaber., et al. "Gender-Related Differences in Acute Aortic Dissection". Circulation 109.24 (2004): 30143021.

7. G Torsello., et al. "S2k guidelines for the diagnosis and treatment of type B aortic dissection". Gefässchirurgie 24.S1 (2019): 19-24.

8. 2014 ESC Guidelines on the diagnosis and treatment of aortic diseases". European Heart Journal 35.41 (2014): 2873-2926.

9. Ijaz A Khan and Chandra K Nair. "Clinical, Diagnostic, and Management Perspectives of Aortic Dissection". Chest 122.1 (2002): 311-328.
10. Gawinecka., et al. "Acute aortic dissection: pathogenesis, risk factors and diagnosis". Swiss Medical Weekly 147 (2007): 4748.

11. Alice Le Huu., et al. "Acute Type A Aortic Dissection". Cardiac Surgery (2020): 475-486.

12. R Scott Mitchell. "Acute aortic dissections with entry tear in the aortic arch: International Registry of Acute Aortic Dissection to the rescue". The Journal of Thoracic and Cardiovascular Surgery 156.5 (2018): 1794.

13. Dolly Thakkar Michael D Dake. "Management of Type B Aortic Dissections: Treatment of Acute Dissections and Acute Complications from Chronic Dissections". Techniques in Vascular and Interventional Radiology 21.3 (2018): 124-130.

\section{Assets from publication with us}

- Prompt Acknowledgement after receiving the article

- Thorough Double blinded peer review

- Rapid Publication

- Issue of Publication Certificate

- High visibility of your Published work

Website: www.actascientific.com/

Submit Article: www.actascientific.com/submission.php

Email us: editor@actascientific.com

Contact us: +919182824667

Citation: Juna Musa., et al. "Case Report "A Dissecting Aneurism of the Aortic Arch at the Left Subclavian Origin Extending to Descending Thoracic and Abdominal Aorta with False and True Lumens"". Acta Scientific Medical Sciences 4.10 (2020): 54-58. 